Pacific

Journal of

Mathematics

\title{
A NOTE ON MINIMAL GRAPHS OVER CERTAIN UNBOUNDED DOMAINS OF HADAMARD MANIFOLDS
}

MiRIAM TELICHEVESKY 


\title{
A NOTE ON MINIMAL GRAPHS OVER CERTAIN UNBOUNDED DOMAINS OF HADAMARD MANIFOLDS
}

\author{
MIRIAM TELICHEVESKY
}

\begin{abstract}
Given an unbounded domain $\Omega$ of a Hadamard manifold $M$, it makes sense to consider the problem of finding minimal graphs with prescribed continuous data on its cone topology boundary, i.e., on its ordinary boundary together with its asymptotic boundary. In this article it is proved that under the hypothesis that the sectional curvature of $M$ is $\leq-1$, this Dirichlet problem is solvable if $\Omega$ satisfies a certain convexity condition at infinity and if $\partial \Omega$ is mean convex. We also prove that mean convexity of $\partial \Omega$ is a necessary condition, extending to unbounded domains some results that are valid on bounded ones.
\end{abstract}

\section{Introduction}

The classical theorem of Jenkins and Serrin on minimal graphs theory, following the works of Bernstein [1910], Haar [1927], Radó [1930] and Finn [1965], states the following.

Theorem 1 [Jenkins and Serrin 1968, Theorem 1]. Let $D \subset \mathbb{R}^{n}$ be a bounded domain whose boundary is of class $C^{2}$. Then the Dirichlet problem for the minimal surface equation in $D$ is well posed for $C^{2}$ boundary data if and only if the mean curvature of $\partial D$ is everywhere nonnegative.

In the last four decades, several works considered problems related to Theorem 1 in distinct directions. Some of them are listed below together with some references.

- Unbounded domains of $\mathbb{R}^{2}$ : [Hwang 1988; Collin and Krust 1991; Sá Earp and Rosenberg 1989; Ripoll and Tomi 2014; Krust 1989; Kuwert 1993; Kutev and Tomi 1998].

- Bounded domains of a Hadamard manifold $M$ : [Folha and Rosenberg 2012; Mazet et al. 2011; Aiolfi et al. 2016]. 
- Asymptotic Dirichlet problems on Hadamard manifolds: [do Espírito Santo et al. 2010; Ripoll and Telichevesky 2015; Gálvez and Rosenberg 2010; Castéras et al. 2013].

- Replace the ambient space $\mathbb{R}^{n+1}$ by the hyperbolic spaces $\mathbb{H}^{n+1}[$ Barbosa and Sá Earp 1998; Guio and Sá Earp 2005; López 2001; Nitsche 2002] or other ambient spaces with a Killing field satisfying certain hypotheses [Alías and Dajczer 2007; Dajczer et al. 2008; 2013]. In this setting it is natural to consider CMC Killing graphs and there is an extensive bibliography on it.

The purpose of this article is to prove that similar existence and nonexistence results remain valid if in Theorem $1, \mathbb{R}^{n}$ is replaced by a Hadamard manifold $M$ with sectional curvature $K_{M} \leq-1$ and the domain $D$ is unbounded and "strictly convex at infinity" (see Definition 4).

Classically, Dirichlet problems on unbounded domains are considered in $\mathbb{R}^{n}$ without prescribed values at infinity. In fact, sometimes the behavior at infinity of bounded solutions is determined by their boundary values. For instance, in $\mathbb{R}^{2}$ it is a consequence of Theorem 2 of [Collin and Krust 1991], which states that if $u$ and $v$ are distinct solutions of the Dirichlet problem in an unbounded domain $U \subset \mathbb{R}^{2}$ which coincide on $\partial U$, then sup $|u-v|$ must have at least logarithmic growth. However, since the manifolds that we consider in this work have sectional curvature $K_{M} \leq-1$, it turns out that the asymptotic boundary of unbounded domains may be "good enough" to prescribe continuous data on them. It therefore makes sense to consider the generalized Dirichlet problem for the minimal hypersurface equation, Problem 2, described in the sequel. In order to state it, let us introduce some useful notations that are not standard.

Throughout this paper $M$ denotes an $m$-dimensional Hadamard manifold, $m \geq 2$, with sectional curvature $K_{M}$ satisfying $K_{M} \leq-1$. The asymptotic boundary $\partial_{\infty} M$ of $M$ is defined by the set of equivalence classes of geodesic rays that stay at finite distance for all time, and it is possible to compactify $M$ by adding $\partial_{\infty} M$ to it. $\bar{M}:=M \cup \partial_{\infty} M$ carries the so-called cone topology (see [Eberlein and O'Neill 1973]), which makes it canonically homeomorphic to a closed ball. If $U \subset \bar{M}$ is any set, we denote by $\bar{U}^{\text {ct }} \subset \bar{M}$ and $\partial^{\text {ct }} U \subset \bar{M}$ its closure and boundary in terms of the cone topology; we also use the notation $\partial_{\infty} U:=\partial^{\text {ct }} U \cap \partial_{\infty} M$.

Problem 2. Let $\Omega \subset M$ be a $C^{2}$ domain of $M$. Given $\varphi \in C\left(\partial^{\text {ct }} \Omega\right)$, find a minimal graph over $\Omega$ that attains $\varphi$ on its boundary, or, equivalently, find a solution of the Dirichlet problem

$$
\left\{\begin{array}{l}
u \in C^{2}(\Omega) \cap C \bar{\Omega}^{\mathrm{ct}}, \\
\mathcal{M}(u):=\operatorname{div}\left(\frac{\nabla u}{\sqrt{1+|\nabla u|^{2}}}\right)=0 \text { in } \Omega, \\
\left.u\right|_{\partial^{\mathrm{c}} \Omega}=\varphi
\end{array}\right.
$$


Concerning the existence, perhaps the main difficulty dealing with unbounded domains is the nonexistence of natural barriers. In general, barriers are constructed using distance functions to a point or to the boundary of the domain, which cannot be adapted directly to points at infinity. Here the geometry of $M$ at infinity plays an important role. For instance, the hyperbolic spaces $\mathbb{\boxplus}^{n}$ have "good geometry" at infinity by the existence of hyperspheres separating points at infinity and having their principal curvatures with the correct sign. The natural way to generalize this fact in order to use the Hessian comparison theorem and adapt barriers of $\mathbb{t}^{n}$ to other Hadamard manifolds is given by the strict convexity condition (SC condition) at infinity, introduced in [Ripoll and Telichevesky 2015]. In that work it is proved that Problem 2 is solvable for $\Omega=M$ (in this case, it is called the asymptotic Dirichlet problem) and any continuous boundary data if $M$ satisfies the SC condition described below.

Definition 3. A Hadamard manifold $M$ is said to satisfy the strict convexity condition at infinity if for all $x \in \partial_{\infty} M$ and all relatively open subsets $\Gamma \subset \partial_{\infty} M$ with $x \in \Gamma$, there exists an open set $V \subset M$ of class $C^{2}$ such that $x$ is an interior point of $\partial_{\infty} V$ (with respect to the induced topology), $\partial_{\infty} V \subset \Gamma$ and $M \backslash V$ is convex.

At this point, it should be mentioned that under the hypothesis $K_{M} \leq-1$, the SC condition is always satisfied by 2-dimensional manifolds, by the rotationally symmetric ones and by those manifolds with controlled decay on sectional curvature (exponential decay) (see also [Ripoll and Telichevesky 2015]). We also should mention that under the same assumption on $K_{M}$, the SC condition is equivalent to the convex conic neighborhood condition presented by H. Choi [1984] in the study of the asymptotic Dirichlet problem with respect to Laplace's operator on Cartan-Hadamard manifolds; the equivalence is a consequence of a lemma of A. Borbély [1998b, Lemma 1]. In fact, both Dirichlet problems are closely related and may be studied together (see also [Ripoll and Telichevesky 2015]).

Contrasting with the existence results under the SC condition, we cite a counterexample constructed by I. Holopainen and J. Ripoll [2015]. In this work the authors present a Hadamard manifold with $K_{M} \leq-1$ that does not admit a solution to the asymptotic Dirichlet problem for the minimal hypersurface equation for any continuous $\varphi \in C\left(\partial_{\infty} M\right)$, although there are bounded nonconstant minimal graphs globally defined on $M$ (see Theorem 1.1 of [Holopainen and Ripoll 2015]). This counterexample proves that the condition $K_{M} \leq-1$ is not sufficient to solve Problem 2 with any continuous boundary data.

Taking into account all these facts, the following definition is natural.

Definition 4. A domain $\Omega \subset \bar{M}$ is strictly convex at infinity if for any $x \in \partial_{\infty} \Omega$ and any relatively open neighborhood $\Gamma \subset \partial^{\text {ct }} \Omega$ of $x$, there exists an open neighborhood $V=V(x, \Gamma, \Omega) \subset \Omega$ of $x$ such that $\bar{V} \cap \partial^{\text {ct }} \Omega \subset \Gamma$ and all the principal curvatures of $\partial V \cap \Omega$, oriented in the direction of $\Omega \backslash V$, are nonnegative. 
Notice that when $\Omega=M$, this definition coincides with the SC condition. With Definition 4 it is now possible to state our main existence result.

Theorem 5. Let $\Omega \subset M$ be a mean convex domain (with respect to the inward orientation) that is strictly convex at infinity. Then Problem 2 is solvable for any continuous boundary data.

Returning our attention to Theorem 1 , when $\Omega \subset \mathbb{R}^{n}$ is bounded, the mean convexity is a necessary condition to the solvability of Problem 2 for any continuous $\varphi$. The second part of this article is dedicated to proving that mean convexity is also necessary in $M$ if we deal with unbounded domains and require boundedness of solutions. In Section 3 we present some necessary lemmata and the proof of the following nonexistence result.

Theorem 6. Let $\Omega \subset \bar{M}$ be a domain and suppose that there exists $y \in \partial \Omega$ such that the mean curvature of $\partial \Omega$ at $y$ (with respect to the inward orientation) satisfies $H(y)<0$. Then there exists a continuous function $\varphi: \partial^{\mathrm{ct}} \Omega \rightarrow \mathbb{R}$ such that Problem 2 is not solvable.

The construction of $\varphi$ depends on two basic ingredients. First of all, on the local aspect concerning the negativity of the mean curvature $H(y)$, it is essential to guarantee that $\varphi(y)$ is bounded by values of the solution on a small sphere centered at $y$, say, on $S_{r}(y) \cap \Omega$. The second essential ingredient is the existence of a bounded barrier in $\Omega \backslash B_{r}(y)$ with some special properties. Similar results outside $\mathbb{R}^{n}$ were proved on bounded domains considering barriers dependent on the diameter of $\Omega$, as in [Nitsche 2002]. Our main improvement is dropping the dependence on the size of the domain.

Combining the results of Theorems 5 and 6, we get:

Theorem 7. Let $\Omega \subset M$ be a domain that is strictly convex at infinity. Then the Dirichlet problem (Problem 2) is solvable for any continuous boundary data if and only if $\Omega$ is mean convex.

It remains an open question what happens if we assume that $\Omega$ is not strictly convex at infinity. We conjecture that in this case it is also possible to construct a continuous function on $\partial^{\text {ct }} \Omega$ for which the Dirichlet problem is not solvable, and therefore strict convexity at infinity is also a necessary condition. Since it deals with nonexistence of solutions in arbitrarily large domains, Theorem 6 may have an important role in the study of this conjecture.

To finish, we should mention that there is a large gap between the behavior of $K_{M}$ at infinity in the cases where Theorem 5 is true and in the ones where it is false. It also remains unknown if there exists some sharp condition on $K_{M}$ that assures solvability of Problem 2 for any continuous boundary data. 


\section{Existence result}

This section is dedicated to proving Theorem 5 . We start with a very important tool, the comparison principle for unbounded domains. It plays an important role in both existence and uniqueness. For now, we just need to work with functions that extend continuously to the asymptotic boundary, however in Section 3 we treat a larger class of functions, as stated above.

Proposition 8 (comparison principle for unbounded domains). Let $U \subset M$ be an unbounded domain of $M$. If $u, v \in C^{2}(U)$ are such that $\mathcal{M}(v) \leq \mathcal{M}(u)$ on $U$ with $\limsup _{p \rightarrow x} u \leq \liminf _{p \rightarrow x} v$ for all $x \in \partial^{\text {ct }} U$, then $u \leq v$ in $U$.

Proof. Choose $o \in M$. Let $\varepsilon>0$ be an arbitrary constant. Using the basis of the cone topology of $\bar{M}$, we obtain that for all $x \in \partial_{\infty} U$, there is an open truncated cone $N_{x}:=T_{o}\left(x, \alpha_{x}, R_{x}\right)$ (that is, the image of a truncated cone of opening angle $\alpha_{x}$ and radius $R_{x}$ by the exponential map of a point $o$ ) such that $u<v+\varepsilon$ on $N_{x}$. Since $\partial_{\infty} U$ is compact, there exists uniform $R$ such that $u<v+\varepsilon$ on $U \backslash B_{R}(o)$. In addition, notice that the hypothesis implies that $u \leq v$ on $\partial U$. Therefore we have $u \leq v+\varepsilon$ on $\partial\left(U \cap B_{R}(o)\right)$, which implies, by the comparison principle on bounded domains, that $u \leq v+\varepsilon$ on $U \cap B_{R}(o)$, and hence the last inequality holds on $U$. Since $\varepsilon$ is arbitrary, the proof is complete.

We now prove Theorem 5 using Perron's method.

A function $\Sigma \in C^{0}\left(\bar{\Omega}^{\mathrm{ct}}\right)$ is called a supersolution for $\mathcal{M}$ if, given a bounded subdomain $U \subset \Omega$, if $u \in C^{2}(U) \cap C^{0}(\bar{U})$ is a solution of $\mathcal{M}=0$ in $U$, the condition $\left.u\right|_{\partial U} \leq\left.\Sigma\right|_{\partial U}$ implies that $u \leq\left.\Sigma\right|_{U}$. A subsolution is defined by replacing $\leq$ by $\geq$.

Let $S_{\varphi}$ be defined by

$$
S_{\varphi}:=\left\{v \in C^{0}\left(\bar{\Omega}^{\mathrm{ct}}\right) \mid v \text { is a subsolution for } \mathcal{M} \text { with }\left.v\right|_{\partial^{\mathrm{ct}} \Omega} \leq \varphi\right\} .
$$

By Proposition $8, v_{0} \equiv \min \varphi \in S_{\varphi}$, which implies that $S_{\varphi} \neq \varnothing$, and $w \equiv \max \varphi$ is such that $v \leq w$ for all $v \in S_{\varphi}$. These facts imply that $u: \Omega \rightarrow \mathbb{R}$ given by

$$
u(x):=\sup \left\{v(x) \mid v \in S_{\varphi}\right\}
$$

is well-defined, and we shall prove that under the hypotheses of Theorem 5, we have $u \in C^{\infty}(\Omega) \cap C\left(\bar{\Omega}^{\mathrm{ct}}\right), \mathcal{M}(u)=0$ and $\left.u\right|_{\partial \mathrm{ct} \Omega}=\varphi$.

We first prove that $u \in C^{\infty}(\Omega)$ and $\mathcal{M}(u)=0$. Given $x \in \Omega$, let $r=r_{x}>0$ be sufficiently small such that the open geodesic ball of center $x$ and radius $r$ satisfies $B_{r}(x) \subset \Omega$ and furthermore $r$ satisfies the inequality

$$
\frac{(n-1)^{2}}{n} \operatorname{coth}^{2} r \geq-\inf _{B_{r}(x)} \operatorname{Ric}_{M} \text {. }
$$

Such $r>0$ exists because coth $r \rightarrow+\infty$ as $r \rightarrow 0^{+}$and Ric $_{M}$ is of course bounded in bounded sets containing $x$. 
The cylinder $\partial B_{r}(x) \times \mathbb{R} \subset M \times \mathbb{R}$ has mean curvature $\geq \frac{n-1}{n}$ coth $r$ (pointing inward) as a consequence of the Hessian comparison theorem, and therefore this choice of $r$ implies, by Theorem 2 of [Dajczer et al. 2013], the existence of minimal graphs in $B_{r}(x)$ extending continuously to any prescribed continuous boundary data on $\partial B_{r}(x)$, and this is an essential fact when we use Perron's method.

Consider a sequence $\left(v_{m}\right)_{m} \subset S_{\varphi}$ such that $\lim _{m} v_{m}(x)=u(x)$. Theorem 2 of [Dajczer et al. 2013] again implies that for each $m \in \mathbb{N}$ there exists a solution $w_{m, x} \in C^{\infty}\left(B_{r}(x)\right) \cap C\left(\overline{B_{r}(x)}\right)$ of $\mathcal{M}=0$ such that $\left.w_{m, x}\right|_{\partial B_{r}(x)}=\left.v_{m}\right|_{\partial B_{r}(x)}$. The interior gradient estimate given by Theorem 1 of [Dajczer et al. 2013] implies that $\left(w_{m, x}\right)_{m}$ contains a subsequence converging uniformly on compact subsets of $B_{r}(x)$ to a solution $w_{x} \in C^{\infty}\left(B_{r}(x)\right)$ of $\mathcal{M}=0$. As in [Gilbarg and Trudinger 1998, Section 2.8], one may prove that $w_{x}=\left.u\right|_{B_{r}(x)}$, which implies that $u \in C^{\infty}(\Omega)$ and $\mathcal{M}=0$. This is done by taking the limit of minimal lifts $u_{m} \in S_{\varphi}$ of each $v_{m}$ defined by

$$
u_{m}(y):= \begin{cases}v_{m}(y) & \text { if } y \in \Omega \backslash B_{r}(x), \\ w_{m, x}(y) & \text { if } y \in B_{r}(x)\end{cases}
$$

We now need to prove that $u$ extends continuously to the desired boundary data on $\partial^{\text {ct }} \Omega$. Since $\partial \Omega$ is mean convex, standard arguments guarantee that the solution assumes the desired data on $\partial \Omega$. To conclude the proof it is necessary to guarantee that it also extends continuously to $\partial_{\infty} \Omega$, hence in the following we construct barriers at infinity.

Given $x \in \partial^{\text {ct }} \Omega$ and an open subset $V$ such that $x \in \partial^{\text {ct }} V \cap \partial^{\text {ct }} \Omega$, we call an upper barrier for $\mathcal{M}$ relative to $x$ and $V$ with height $C$ a function $\Sigma \in C(\Omega)$ such that

(i) $\Sigma$ is a supersolution for $\mathcal{M}$;

(ii) $\Sigma \geq 0$ and $\lim _{p \in \Omega, p \rightarrow x} \Sigma(p)=0$, the limit with respect to the cone topology;

(iii) $\Sigma_{\Omega \backslash V} \geq C$.

Lower barriers are defined analogously.

A point $x \in \partial^{\mathrm{ct}} \Omega$ is said to be regular (with respect to the mean curvature operator $\mathcal{M}$ ) if it satisfies the following property: given $C>0$ and a relatively open subset $\Gamma \subset \partial^{\text {ct }} \Omega$ with $x \in \Gamma$, there exist an open set $V \subset \Omega$ such that $x$ is an interior point of $\bar{V} \cap \partial^{\text {ct }} \Omega$ (with respect to the topology induced on the boundary), with $\bar{V} \cap \partial^{\text {ct }} \Omega \subset \Gamma$, and an upper barrier $\Sigma: \Omega \rightarrow \mathbb{R}$ relative to $x$ and $V$ with height $C$.

The following lemma is analogous to Theorem 2.7 of [Choi 1984], but we present the proof for the sake of completeness.

Lemma 9. The function $u$ given by (1) extends continuously to $\varphi$ to each regular point $x \in \partial_{\infty} \Omega$.

Proof. Given $x \in \partial_{\infty} \Omega$ and $\varepsilon>0$, let $\Gamma \subset \partial^{\text {ct }} \Omega$ be such that $|\varphi-\varphi(x)|<\varepsilon / 2$ in $\Gamma$. Let $\Sigma: \Omega \rightarrow \mathbb{R}$ be an upper barrier relative to $x$ and $V$ with height $C=\max |\varphi|$, 
where $V$ is given by the definition of regularity. It follows that $w:=\Sigma+\varphi(x)+\varepsilon$ is a supersolution for $\mathcal{M}$. By the choice of $\Gamma$, it holds that $w>\varphi$ on $\Gamma$ and since $\left.w\right|_{\partial^{\mathrm{ct}} \Omega \backslash \Gamma} \geq \max |\varphi|$, it of course satisfies $w \geq \varphi$ on $\partial^{\mathrm{ct}} \Omega \backslash \Gamma$. Therefore $v \leq w$ for all $v \in S_{\varphi}$, which implies that

$$
\lim _{p \in \Omega, p \rightarrow x} u(p) \leq \lim _{p \in \Omega, p \rightarrow x} w(p)=\varphi(x)+\varepsilon .
$$

On the other hand, notice that $v_{0}:=\varphi(x)-\varepsilon-\Sigma$ belongs to $S_{\varphi}$ and therefore $u \geq v_{0}$ in $\Omega$, which implies that

$$
\lim _{p \in \Omega, p \rightarrow x} u(p) \geq \lim _{p \in \Omega, p \rightarrow x} v_{0}(p)=\varphi(x)-\varepsilon .
$$

Since $\varepsilon$ is arbitrary, we have $\varphi(x) \leq \lim _{p \in \Omega, p \rightarrow x} u(p) \leq \varphi(x)$.

To finish, we now prove regularity at the points of $\partial_{\infty} \Omega$.

Proposition 10. Let $\Omega \subset M$ be a domain that is strictly convex at infinity. Then $\mathcal{M}$ is regular at each point of $\partial_{\infty} \Omega$.

Proof. Let $x \in \partial_{\infty} \Omega$ and let $\Gamma \subset \partial^{\text {ct }} \Omega$ be a relatively open neighborhood of $x$. By hypothesis, there exists an open neighborhood $V \subset \Omega$ of $x$ such that $\bar{V} \cap \partial^{\text {ct }} \Omega \subset \Gamma$ and $\partial V \cap \Omega$ has nonnegative principal curvatures.

Let $s: V \rightarrow \mathbb{R}$ be the distance function to $\partial V \cap \Omega$. Since $K_{M} \leq-1$ and all principal curvatures of $\partial V \cap \Omega$ are nonnegative, we have that the Laplacian of $s$ satisfies

$$
\Delta s \geq(n-1) \tanh s
$$

(see, for instance, Theorem 4.3 of [Choi 1984]).

Define $g:(0,+\infty) \rightarrow \mathbb{R}$ by

$$
g(s):=\int_{s}^{+\infty} \frac{d t}{\sqrt{\cosh ^{2(n-1)} t-1}} .
$$

Notice that $g$ is well-defined and $\lim _{s \rightarrow 0^{+}} g(s)=+\infty, \lim _{s \rightarrow+\infty} g(s)=0$. Define now $w: V \rightarrow \mathbb{R}$ by $w(p):=g(s(p))$. A straightforward computation gives

$$
\mathcal{M}(w)=(n-1) \cosh ^{n-1} s \sinh s+(1-n) \cosh ^{1-n} s \Delta s
$$

and hence the estimate $\Delta s \geq \tanh s$ leads to $\mathcal{M}(w) \leq 0$.

We remark that $w$ is a solution if $M=\mathbb{H}^{n}$ and $V$ is a totally geodesic hypersphere.

To finish with the proof, define the supersolution $\Sigma \in C^{0}(\bar{\Omega})$ by

$$
\Sigma(p)= \begin{cases}\min \{w(p), C\} & \text { if } p \in V, \\ C & \text { if } p \in \bar{\Omega} \backslash V,\end{cases}
$$

which is of course an upper barrier relative to $x$ and $V$ with height $C$, and hence the proof is complete. 


\section{Nonexistence result}

We now prove that mean convexity of $\partial \Omega$ is a necessary condition, as stated in Theorem 6. We start with the next classical lemma, proved by Jenkins and Serrin [1968] in the case where the domain is bounded and contained on $\mathbb{R}^{n}$.

Lemma 11. Let $U \subset M$ be an open domain and $\Gamma$ a relatively $C^{1}$ open subset of $\partial U$. If $u \in C(\bar{U}) \cap C^{2}(U \cup \Gamma)$ and $w \in C(\bar{U}) \cap C^{2}(U)$ satisfy

$$
\begin{aligned}
\mathcal{M}(w) & <\mathcal{M}(u) & & \text { in } U, \\
u & \leq w & & \text { on } \partial U \backslash \Gamma, \text { and } \\
\frac{\partial w}{\partial v} & =-\infty & & \text { in } \Gamma,
\end{aligned}
$$

where $v$ is the inner normal vector to $\partial U$, then $u \leq w$ in $U$.

Proof. If $u \leq w$ on $\Gamma$, the result is a consequence of the comparison principle. Suppose, towards a contradiction, that there exists $y \in \operatorname{Int} \Gamma$ such that

$$
d:=\max _{\Gamma}(u-w)=u(y)-w(y)>0 .
$$

Then $u \leq w+d$ on $\partial U$, and hence by the comparison principle we have $u \leq w+d$ in $U$. Therefore

$$
\frac{\partial}{\partial v}(u-w)(y) \leq 0 \Rightarrow \frac{\partial}{\partial v}(u)(y) \leq-\infty,
$$

contradicting the fact that $u \in C^{2}(U \cup \Gamma)$.

Lemma 12. Let $\Omega \subset M$ be an open $C^{2}$ domain (possibly unbounded) with mean curvature (with respect to the inner normal) $H: \partial \Omega \rightarrow \mathbb{R}$. Suppose that there exist $y \in \partial \Omega$ such that $H(y)<0$. Then there exists $s>0$ depending only on the local geometry of $\Omega$ near $y$ and $C>0$ depending only on $H(y)$ such that if $u \in C^{2}(\bar{\Omega}) \cap C\left(\bar{\Omega}^{\mathrm{ct}}\right)$ satisfy $\mathcal{M}(u)=0$ in $\Omega$, then

$$
u(y) \leq C+\sup _{\partial B_{s}(y) \cap \Omega} u .
$$

Proof. Let $d: \widetilde{\Omega} \rightarrow \mathbb{R}$ be given by $d(x)=\operatorname{dist}(x, \partial \Omega)$, where $\widetilde{\Omega} \subset \Omega$ is the open subset where $d$ is smooth. Since $H(y)<0$, it holds that $\Delta d(y)=-H(y)>0$. Since $\partial \Omega$ is $C^{2}$, there exists $s>0$ such that $B_{s}(y) \cap \Omega \subset \widetilde{\Omega}$ and

$$
\Delta d(x)>-\frac{H(y)}{2}=: \epsilon, \quad \forall x \in B_{s}(y) \cap \Omega .
$$

This is the required $s$.

We claim that if $x \in B_{s}(y) \cap \Omega$, then $u(x) \leq C+\sup _{\partial B_{s}(y) \cap \Omega} u$. To prove it, let $\Gamma_{x}$ be the level set of $d$ that contains $x$ and $\Omega_{x}$ be the set enclosed by $\Gamma_{x}$ and $\partial B_{s}(y)$, that is, $\Omega_{x}:=\left\{p \in B_{s}(y) \mid d(p)>d(x)\right\}$. 
Consider $\psi$ given by

$$
\psi(t)=\frac{\pi}{2}-\operatorname{arcsec}(t+1) .
$$

Then $\psi \geq 0, \psi(0)=\pi / 2$ and $\lim _{t \rightarrow+\infty} \psi(t)=0$. Its first and second derivatives are given below:

$$
\begin{aligned}
\psi^{\prime}(t) & =-\frac{1}{(t+1) \sqrt{t^{2}+2 t}}, \\
\psi^{\prime \prime}(t) & =\frac{1}{(t+1)^{2} \sqrt{t^{2}+2 t}}+\frac{1}{\left(t^{2}+2 t\right)^{3 / 2}} .
\end{aligned}
$$

Define $w: B_{s}(y) \cap \Omega_{x} \rightarrow \mathbb{R}$ by

$$
w(p):=A \psi(d(p))+\sup _{\partial B_{s}(y) \cap \Omega} u,
$$

where $A>0$ is to be determined. After some computations we obtain

$$
\left(1+|\nabla w|^{2}\right)^{3 / 2} \mathcal{M}(w)(p)=A \psi^{\prime \prime}(d(p))+\left(A \psi^{\prime}(d(p))+A^{3} \psi^{\prime}(d(p))^{3}\right) \Delta d(p) .
$$

Using then that $\Delta d(p)>\epsilon$ and $\psi^{\prime}<0$ in the domain we are considering, we obtain

$$
\begin{aligned}
\left(1+|\nabla w|^{2}\right)^{3 / 2} \mathcal{M}(w) & \leq A\left[\psi^{\prime \prime}+\epsilon \psi^{\prime}+\epsilon A^{2} \psi^{\prime 3}\right] \\
& =A(t+1)^{-3}\left(t^{2}+2 t\right)^{-3 / 2}\left[(t+1)\left(t^{2}+2 t\right)+(t+1)^{3}\right. \\
& \left.-\epsilon(t+1)^{2}\left(t^{2}+2 t\right)-\epsilon A^{2}\right] .
\end{aligned}
$$

Notice that the term in the brackets is a polynomial of degree 4 with leading coefficient $-\epsilon<0$ and constant term $1-\epsilon A^{2}$. Then it is clear that there exists $A>0$ large enough that this polynomial is negative for all $t \geq 0$; with this choice of $A$ we obtain that $\mathcal{M}(w)<0$ on $\Omega_{x}$.

Furthermore, by definition of $w$ we have $w \geq u$ on $\partial B_{s}(y) \cap \Omega_{x}$ and $\partial w / \partial v=-\infty$ on $\Gamma_{x}$, which is an open $C^{1}$ portion of $\partial \Omega_{x}$. We also notice that $u \in C^{2}\left(\Gamma_{x}\right)$. By Lemma 11 , we obtain $w \geq u$ in $\Omega_{x}$. Since $x$ is arbitrary and $u$ is continuous, it holds the desired inequality with $C=A \frac{\pi}{2}$, which concludes the proof.

Proposition 13. Let $M$ be a Hadamard manifold with sectional curvature $K_{M} \leq-1$. There exists universal $C>0$ such that if $\Omega$ is a $C^{1}$ domain of $M$ and $u$ satisfies $\mathcal{M}(u)=0$ in $\Omega$, then

$$
\sup _{\partial B_{s}(y) \cap \Omega} u \leq C+\sup _{\partial^{\mathrm{ct}} \Omega \backslash B_{s}(y)} u
$$

for all $y \in \partial \Omega$ and $s>0$ such that $\partial B_{s}(y) \cap \Omega$ is a nonempty connected set.

Proof. Consider $w: \Omega \backslash B_{s}(y) \rightarrow \mathbb{R}$ given by

$$
w(x)=B \psi(r(x))+\sup _{\partial^{\mathrm{ct}} \Omega \backslash B_{s}(y)} u,
$$


where $\psi$ is given by $(6), r(x):=\operatorname{dist}\left(x, \partial B_{s}(y)\right)$ and $B$ is an appropriate constant to be chosen latter. Since $K_{M} \leq-1$, we have by the Hessian comparison theorem that $\Delta r \geq n-1$. Hence, mimicking the computations of the previous lemma, we obtain the same polynomial, except that we have $n-1$ instead of $\epsilon$ and $B$ instead of $A$. It is again clear that there exists $B$ large enough that $\mathcal{M}(w) \leq 0$. We remark that such a constant does not depend on anything (except in the fact that $K_{M} \leq-1$ ) since we may choose the constant that is appropriate to the case $n=2$ and it works on all dimensions.

We are again in the situation of the hypotheses of Lemma 11, with $U=\Omega \backslash B_{s}(y)$. Hence we obtain, for all $x \in \partial B_{s}(y) \cap \Omega$,

$$
u(x) \leq \sup _{\partial^{\mathrm{ct}} \Omega \backslash B_{s}(y)} u+B \frac{\pi}{2}
$$

and the proof is complete.

Proof of Theorem 6. By combining the estimates obtained in Lemma 12 and Proposition 13, we obtain the existence of a continuous function $\varphi: \partial \Omega \rightarrow \mathbb{R}$ for which Problem 2 is not solvable: it suffices to put $\varphi(y)=\pi(A+B)$, where $A$ and $B$ are given by the previous results, and $\varphi=0$ on $\partial \Omega \backslash B_{s}(y)$, where $s$ is given in the proof of Lemma 12.

\section{Applications}

Corollary 14. Let $\Omega$ be a domain that has only finitely many points on $\partial_{\infty} \Omega$. Then Problem 2 is solvable for any continuous $\varphi$ if and only if $\Omega$ is mean convex.

Proof. Notice that since $\partial_{\infty} M$ is compact, $\partial_{\infty} \Omega$ is also compact and therefore "finitely many points on $\partial_{\infty} \Omega$ " is equivalent to "isolated points on $\partial_{\infty} \Omega$ ". In order to apply Theorem 7 , it suffices to prove that $\Omega$ is strictly convex at infinity.

Given $x \in \partial_{\infty} \Omega$, let $W \subset \bar{\Omega}^{\text {ct }}$ be a relatively open neighborhood of $x$. We may suppose without loss of generality that $x$ is the only point at infinity of $W$, otherwise we just work with any open subset of $W$ where this property holds. Choosing $o \in M \backslash W$, we have that $W$ is contained on some truncated cone centered at $o$ with radius $R>0$, and as a consequence we have $\partial W \subset M \backslash B_{R}(o)$. Set $V:=\Omega \backslash \overline{B_{R}(o)}$, and it is clear that it satisfies the required conditions.

Corollary 15. If $M$ satisfies the SC condition and $\Omega$ is a mean convex domain of $M$ such that $\partial_{\infty} \Omega$ is composed only of open portions and isolated points, then Problem 2 is solvable in $\Omega$. In particular, this is the case if either $\operatorname{dim} M=2$ or $M$ is rotationally symmetric, or

(7) $\min \left\{K_{M}(\Pi) \mid \Pi\right.$ is a 2-plane in $\left.T_{p} M, p \in B_{R+1}(o)\right\} \geq-\frac{e^{2 k R}}{R^{2+2 \epsilon}}, \quad R \geq R^{*}$ for some constants $\epsilon, R^{*}>0$. 
Particular cases of Corollary 15 may be found in [Ripoll and Telichevesky 2015].

4.1. Application of the technique: Dirichlet problems for p-Laplacians. Consider now the following Dirichlet problem for the $p$-Laplacian operator, $p>1$, for continuous $u$ in the Sobolev space $W^{1, p}(\Omega)$ :

$$
\left\{\begin{array}{l}
\Delta_{p}(u):=\operatorname{div}\left(|\nabla u|^{p-2} \nabla u\right)=0 \quad \text { in } \Omega \\
\left.u\right|_{\partial \Omega}=\varphi
\end{array}\right.
$$

Concerning the case $\Omega=M$, the counterexamples constructed by A. Ancona [1994] and by A. Borbély [1998a] show that some convexity at infinity is also needed to obtain existence of solutions of asymptotic Dirichlet problems related to the Laplacian operator $\Delta$. I. Holopainen [2015] constructed a counterexample for the $p$-Laplacian operator $\Delta_{p}$. The manifolds constructed by them contain a point in $\partial_{\infty} M$ with the property that any open neighborhood of it has the whole manifold as the convex hull, and hence $M$ is not strictly convex at infinity.

On the other hand, in [Ripoll and Telichevesky 2015] the authors proved that the SC condition is sufficient for solvability of asymptotic Dirichlet problems with respect to $\Delta_{p}$. We may extend this result to our case, proving that if $\Omega$ is strictly convex at infinity, then every $x \in \partial_{\infty} \Omega$ is regular with respect to the operator $\Delta_{p}$.

The proof is mutatis mutandis the same as we have done above; it is sufficient to replace $\mathcal{M}$ by $\Delta_{p}$ and the function $g$ constructed in Proposition 10 by

$$
g(s):=c \int_{s}^{+\infty} \cosh ^{(1-n) /(p-1)}(t) d t,
$$

where $c$ is a sufficiently large constant $\left(c=2 C(\cosh 1)^{(n-1) /(p-1)}\right.$ works).

Together with the classical theory of existence of solutions over bounded domains that satisfy the exterior sphere condition, we obtain the following result.

Theorem 16. Let $M$ be a Hadamard manifold with sectional $K_{M} \leq-1$. Let $\Omega \subset M$ be an unbounded domain that is strictly convex at infinity and that satisfies the exterior sphere condition on its finite part, namely, given $x \in \partial \Omega$, there exist a sphere contained in $M \backslash \Omega$ that is tangent to $\partial \Omega$ at $x$. Then (8) is solvable for any $\varphi \in C\left(\partial^{\mathrm{ct}} \Omega\right)$.

\section{Acknowledgment}

We would like to thank the referee for valuable remarks and questions.

\section{References}

[Aiolfi et al. 2016] A. Aiolfi, J. B. Ripoll, and M. Soret, "The Dirichlet problem for the minimal hypersurface equation on arbitrary domains of a Riemannian manifold", Manuscripta Math. 149:1-2 (2016), 71-81. MR 3447141 
[Alías and Dajczer 2007] L. J. Alías and M. Dajczer, "Normal geodesic graphs of constant mean curvature", J. Differential Geom. 75:3 (2007), 387-401. MR 2007m:53005 Zbl 1119.53040

[Ancona 1994] A. Ancona, "Convexity at infinity and Brownian motion on manifolds with unbounded negative curvature”, Rev. Mat. Iberoam. 10:1 (1994), 189-220. MR 95a:58132 Zbl 0804.58056

[Barbosa and Sá Earp 1998] J. L. M. Barbosa and R. Sá Earp, "Prescribed mean curvature hypersurfaces in $H^{n+1}(-1)$ with convex planar boundary, I", Geom. Dedicata 71:1 (1998), 61-74. MR 99d:53064 Zbl 0922.53023

[Bernstein 1910] S. Bernstein, "Sur les surfaces définies au moyen de leur courbure moyenne ou totale”, Ann. Sci. École Norm. Sup. (3) 27 (1910), 233-256. MR 1509123 JFM 41.0692.05

[Borbély 1998a] A. Borbély, "The nonsolvability of the Dirichlet problem on negatively curved manifolds”, Differential Geom. Appl. 8:3 (1998), 217-237. MR 99j:53043 Zbl 0947.53019

[Borbély 1998b] A. Borbély, "Some results on the convex hull of finitely many convex sets", Proc. Amer. Math. Soc. 126:5 (1998), 1515-1525. MR 98j:53039 Zbl 0902.53030

[Castéras et al. 2013] J.-B. Castéras, I. Holopainen, and J. B. Ripoll, "On the asymptotic Dirichlet problem for the minimal hypersurface equation in a Hadamard manifold", preprint, 2013. arXiv $1311.5693 \mathrm{v} 1$

[Choi 1984] H. I. Choi, "Asymptotic Dirichlet problems for harmonic functions on Riemannian manifolds”, Trans. Amer. Math. Soc. 281:2 (1984), 691-716. MR 85b:53040 Zbl 0541.53035

[Collin and Krust 1991] P. Collin and R. Krust, "Le problème de Dirichlet pour l'équation des surfaces minimales sur des domaines non bornés”, Bull. Soc. Math. France 119:4 (1991), 443-462. MR 92m:53007 Zbl 0754.53013

[Dajczer et al. 2008] M. Dajczer, P. A. Hinojosa, and J. H. de Lira, "Killing graphs with prescribed mean curvature”, Calc. Var. Partial Differential Equations 33:2 (2008), 231-248. MR 2009m:53154 Zbl 1152.53046

[Dajczer et al. 2013] M. Dajczer, J. H. de Lira, and J. B. Ripoll, "An interior gradient estimate for the mean curvature equation of Killing graphs and applications", preprint, 2013. To appear in J. Anal. Math. arXiv 1206.2900

[Eberlein and O'Neill 1973] P. Eberlein and B. O'Neill, "Visibility manifolds", Pacific J. Math. 46 (1973), 45-109. MR 49 \#1421 Zbl 0264.53026

[do Espírito Santo et al. 2010] N. do Espírito Santo, S. Fornari, and J. B. Ripoll, "The Dirichlet problem for the minimal hypersurface equation in $\mathbb{M} \times \mathbb{R}$ with prescribed asymptotic boundary", $J$. Math. Pures Appl. (9) 93:2 (2010), 204-221. MR 2011e:35138 Zbl 1193.58012

[Finn 1965] R. Finn, "Remarks relevant to minimal surfaces, and to surfaces of prescribed mean curvature", J. Analyse Math. 14 (1965), 139-160. MR 32 \#6337 Zbl 0163.34604

[Folha and Rosenberg 2012] A. Folha and H. Rosenberg, "The Dirichlet problem for constant mean curvature graphs in $\mathbb{M} \times \mathbb{R} "$, Geom. Topol. 16:2 (2012), 1171-1203. MR 2946806 Zbl 1281.53013

[Gálvez and Rosenberg 2010] J. A. Gálvez and H. Rosenberg, "Minimal surfaces and harmonic diffeomorphisms from the complex plane onto certain Hadamard surfaces", Amer. J. Math. 132:5 (2010), 1249-1273. MR 2011j:53012 Zbl 1229.53064

[Gilbarg and Trudinger 1998] D. Gilbarg and N. S. Trudinger, Elliptic partial differential equations of second order, revised 2nd ed., Grundlehren der Mathematischen Wissenschaften 224, Springer, Berlin, 1998. MR 2001k:35004 Zbl 1042.35002

[Guio and Sá Earp 2005] E. M. Guio and R. Sá Earp, "Existence and non-existence for a mean curvature equation in hyperbolic space", Commun. Pure Appl. Anal. 4:3 (2005), 549-568. MR 2006i:35093 Zbl 1106.35014

[Haar 1927] A. Haar, "Über das Plateausche Problem”, Math. Ann. 97:1 (1927), 124-158. MR 1512358 JFM 52.0710.02 
[Holopainen 2015] I. Holopainen, "Nonsolvability of the asymptotic Dirichlet problem for the p-Laplacian on Cartan-Hadamard manifolds", Adv. Calc. Var. (online publication February 2015).

[Holopainen and Ripoll 2015] I. Holopainen and J. B. Ripoll, "Nonsolvability of the asymptotic Dirichlet problem for some quasilinear elliptic PDEs on Hadamard manifolds", Rev. Mat. Iberoam. 31:3 (2015), 1107-1129. MR 3420486 Zbl 06503648

[Hwang 1988] J.-F. Hwang, "Comparison principles and Liouville theorems for prescribed mean curvature equations in unbounded domains", Ann. Scuola Norm. Sup. Pisa Cl. Sci. (4) 15:3 (1988), 341-355. MR 90m:35019 Zbl 0705.49022

[Jenkins and Serrin 1968] H. Jenkins and J. Serrin, "The Dirichlet problem for the minimal surface equation in higher dimensions", J. Reine Angew. Math. 229 (1968), 170-187. MR 36 \#5519 Zbl 0159.40204

[Krust 1989] R. Krust, "Remarques sur le problème extérieur de Plateau", Duke Math. J. 59:1 (1989), 161-173. MR 90i:49050 Zbl 0709.49022

[Kutev and Tomi 1998] N. Kutev and F. Tomi, "Existence and nonexistence for the exterior Dirichlet problem for the minimal surface equation in the plane", Differential Integral Equations 11:6 (1998), 917-928. MR 99m:35062 Zbl 1074.35550

[Kuwert 1993] E. Kuwert, "On solutions of the exterior Dirichlet problem for the minimal surface equation”, Ann. Inst. H. Poincaré Anal. Non Linéaire 10:4 (1993), 445-451. MR 94i:35034 Zbl 0820.35038

[López 2001] R. López, "Graphs of constant mean curvature in hyperbolic space", Ann. Global Anal. Geom. 20:1 (2001), 59-75. MR 2002e:53009 Zbl 0996.53039

[Mazet et al. 2011] L. Mazet, M. M. Rodríguez, and H. Rosenberg, "The Dirichlet problem for the minimal surface equation, with possible infinite boundary data, over domains in a Riemannian surface”, Proc. Lond. Math. Soc. (3) 102:6 (2011), 985-1023. MR 2012f:53013 Zbl 1235.53007

[Nitsche 2002] P.-A. Nitsche, "Existence of prescribed mean curvature graphs in hyperbolic space", Manuscripta Math. 108:3 (2002), 349-367. MR 2003f:53015 Zbl 1130.53302

[Radó 1930] T. Radó, "The problem of the least area and the problem of Plateau”, Math. Z. 32:1 (1930), 763-796. MR 1545197 JFM 56.0436.01

[Ripoll and Telichevesky 2015] J. B. Ripoll and M. Telichevesky, "Regularity at infinity of Hadamard manifolds with respect to some elliptic operators and applications to asymptotic Dirichlet problems", Trans. Amer. Math. Soc. 367:3 (2015), 1523-1541. MR 3286491 Zbl 1308.58011

[Ripoll and Tomi 2014] J. B. Ripoll and F. Tomi, "On solutions to the exterior Dirichlet problem for the minimal surface equation with catenoidal ends", Adv. Calc. Var. 7:2 (2014), 205-226. MR 3187916 Zbl 1292.35110

[Sá Earp and Rosenberg 1989] R. Sá Earp and H. Rosenberg, "The Dirichlet problem for the minimal surface equation on unbounded planar domains”, J. Math. Pures Appl. (9) 68:2 (1989), 163-183. MR 90m:35072 Zbl 0696.49069

Received February 5, 2015. Revised September 17, 2015.

MiRIAM TELICHEVESKY

INSTITUTO DE MATEMÁTICA

UNIVERSIDADE FEDERAL DO RIO GRANDE DO SUL

91509-900 Porto ALEGRE, RS

BRAZIL

miriam.telichevesky@ufrgs.br 


\title{
PACIFIC JOURNAL OF MATHEMATICS
}

\author{
msp.org/pjm
}

Founded in 1951 by E. F. Beckenbach (1906-1982) and F. Wolf (1904-1989)

\section{EDITORS}

Don Blasius (Managing Editor)

Department of Mathematics

University of California

Los Angeles, CA 90095-1555

blasius@math.ucla.edu

\author{
Paul Balmer \\ Department of Mathematics \\ University of California \\ Los Angeles, CA 90095-1555 \\ balmer@math.ucla.edu \\ Robert Finn \\ Department of Mathematics \\ Stanford University \\ Stanford, CA 94305-2125 \\ finn@math.stanford.edu \\ Sorin Popa \\ Department of Mathematics \\ University of California \\ Los Angeles, CA 90095-1555 \\ popa@math.ucla.edu
}

\author{
Vyjayanthi Chari \\ Department of Mathematics \\ University of California \\ Riverside, CA 92521-0135 \\ chari@math.ucr.edu \\ Kefeng Liu \\ Department of Mathematics \\ University of California \\ Los Angeles, CA 90095-1555 \\ liu@math.ucla.edu \\ Jie Qing \\ Department of Mathematics \\ University of California \\ Santa Cruz, CA 95064 \\ qing@ cats.ucsc.edu
}

\section{PRODUCTION}

Silvio Levy, Scientific Editor, production@msp.org

\section{SUPPORTING INSTITUTIONS}

ACADEMIA SINICA, TAIPEI

CALIFORNIA INST. OF TECHNOLOGY

INST. DE MATEMÁTICA PURA E APLICADA

KEIO UNIVERSITY

MATH. SCIENCES RESEARCH INSTITUTE

NEW MEXICO STATE UNIV.

OREGON STATE UNIV.

\author{
STANFORD UNIVERSITY \\ UNIV. OF BRITISH COLUMBIA \\ UNIV. OF CALIFORNIA, BERKELEY \\ UNIV. OF CALIFORNIA, DAVIS \\ UNIV. OF CALIFORNIA, LOS ANGELES \\ UNIV. OF CALIFORNIA, RIVERSIDE \\ UNIV. OF CALIFORNIA, SAN DIEGO \\ UNIV. OF CALIF., SANTA BARBARA
}

\author{
Daryl Cooper \\ Department of Mathematics \\ University of California \\ Santa Barbara, CA 93106-3080 \\ cooper@math.ucsb.edu \\ Jiang-Hua Lu \\ Department of Mathematics \\ The University of Hong Kong \\ Pokfulam Rd., Hong Kong \\ jhlu@maths.hku.hk \\ Paul Yang \\ Department of Mathematics \\ Princeton University \\ Princeton NJ 08544-1000 \\ yang@math.princeton.edu
}

These supporting institutions contribute to the cost of publication of this Journal, but they are not owners or publishers and have no responsibility for its contents or policies.

See inside back cover or msp.org/pjm for submission instructions.

The subscription price for 2016 is US $\$ 440 /$ year for the electronic version, and $\$ 600 /$ year for print and electronic.

Subscriptions, requests for back issues and changes of subscribers address should be sent to Pacific Journal of Mathematics, P.O. Box 4163, Berkeley, CA 94704-0163, U.S.A. The Pacific Journal of Mathematics is indexed by Mathematical Reviews, Zentralblatt MATH, PASCAL CNRS Index, Referativnyi Zhurnal, Current Mathematical Publications and Web of Knowledge (Science Citation Index).

The Pacific Journal of Mathematics (ISSN 0030-8730) at the University of California, c/o Department of Mathematics, 798 Evans Hall \#3840, Berkeley, CA 94720-3840, is published twelve times a year. Periodical rate postage paid at Berkeley, CA 94704, and additional mailing offices. POSTMASTER: send address changes to Pacific Journal of Mathematics, P.O. Box 4163, Berkeley, CA 94704-0163.

PJM peer review and production are managed by EditFLOW ${ }^{\circledR}$ from Mathematical Sciences Publishers.

\section{PUBLISHED BY}

\section{mathematical sciences publishers \\ nonprofit scientific publishing}

http://msp.org/

(C) 2016 Mathematical Sciences Publishers 


\section{PACIFIC JOURNAL OF MATHEMATICS}

Volume $281 \quad$ No. $1 \quad$ March 2016

Compatible systems of symplectic Galois representations and the inverse Galois problem II: Transvections and huge image

SARA ARIAS-DE-REYNA, LUIS DIEULEFAIT and GABOR

WIESE

On the number of lines in the limit set for discrete subgroups of $\operatorname{PSL}(3, \mathbb{C})$

Waldemar Barrera, Angel CANo and JuÁn NaVArRete Galois theory, functional Lindemann-Weierstrass, and Manin maps

DANIEL BERTRAND and ANAND PILLAY

Morse area and Scharlemann-Thompson width for hyperbolic

3-manifolds

DiANE HOFFOSS and JOSEPH MAHER

Ricci tensor of real hypersurfaces

MAYUKO KON

Monotonicity formulae and vanishing theorems

JINTANG LI

Jet schemes of the closure of nilpotent orbits

ANNE MOREAU and RuPERT WeI TZE Yu

Components of spaces of curves with constrained curvature on flat surfaces

Nicolau C. Saldanha and Pedro ZüHlke A note on minimal graphs over certain unbounded domains of Hadamard manifolds

MiRIAM TELICHEVESKY 